\title{
Choriorétinite toxoplasmique active de l'œil droit chez une adolescente à propos d'un cas
}

\section{Active toxoplasmic chorioretinitis of the right eye in an adolescent about a case}

\author{
DIALLO $\mathbf{S}^{\mathbf{1}^{*}}$ \\ 1= Institut d'ophtalmologie tropicale d'Afrique (Bamako). \\ Correspondance : Dr Seydou Diallo E-mail : dial1907@yahoo.fr
}

\section{RESUME :}

La choriorétinite toxoplasmique active constitue une affection rare chez les adolescents. Le diagnostic est principalement basé sur l'examen ophtalmologique et une réponse favorable au traitement. Nous rapportons le cas d'une adolescente de 18 ans reçue pour une vision floue avec douleur oculaire. L'examen du segment antérieur est sans particularité. Le fond d'œil au niveau de l'œil droit retrouve un foyer de choriorétinite toxoplasmique active. L'examen ophtalmologique de l'œil gauche est sans particularité. Le bilan para clinique effectué chez la patiente montre un test de REMINGTON positif à 2 reprises distants de 3 semaines. L'évolution clinique après l'instauration du traitement était favorable avec une cicatrisation du foyer actif en un mois.

MOTS CLES : adolescent, choriorétinite active, toxoplasmique.

\begin{abstract}
:
Toxoplasma active chorioretinitis is a rare condition in adolescents. The diagnosis is mainly based on ophthalmological examination and a favorable response to treatment. We report the case of an 18year-old girl received for blurred vision with eye pain. The examination of the anterior segment is without particularity. The fundus at the level of the right eye found a focus of active toxoplasmic chorioretinitis. The ophthalmological examination of the left eye is without particularity. Paraclinical assessment performed in the patient shows a positive REMINGTON test 2 times 3 weeks apart. The clinical course after initiation of treatment was favorable with healing of the active focus in one month.
\end{abstract}

KEY WORDS: adolescent, active chorioretinitis, toxoplasmic.

\section{INTRODUCTION:}

La toxoplasmose oculaire est une infection parasitaire due au protozoaire toxoplasmose gondii, à multiplication intracellulaire obligatoire, première cause d'uvéite postérieure dans le monde $[1,2]$. La choriorétinite toxoplasmique constitue une des causes de baisse de l'acuité visuelle pouvant survenir à tout âge. Elle se manifeste typiquement sous forme de foyers rétinochoroidiens. Nous présentons un cas de choriorétinite active au niveau de l'œil droit observé au CHU- IOTA.

\section{CAS CLINIQUE :}

Une patiente de 18 ans sans antécédent médical particulier, avec un bon état général a été admise pour la première fois à l'institut d'ophtalmologie tropicale de l'Afrique pour une vision floue avec douleur oculaire depuis 5 jours. L'examen ophtalmologique comprenait au niveau de l'œil droit: La mesure de l'acuité visuelle sur l'échelle de Monnoyer qui avait donné une diminution de l'acuité visuelle à l'œil droit chiffrée à compte les doigts à 5 mètres. L'examen des annexes était normal. L'examen à la lampe à fente : le segment antérieur était sans particularité. La prise de la pression intraoculaire au tonomètre à l'aplanation était chiffrée à $12 \mathrm{~mm}$ de mercure. L'examen du fond d'œil par la lentille de volk 90 a permis de détecter une lésion blanchâtre, ronde, profonde, à bords flou d'un diamètre papillaire de siège temporal supérieur à un et demi de diamètre papillaire de la macula accompagnée d'une tache hémorragique rétinienne au voisinage du foyer. L'examen ophtalmologique de l'œil gauche est sans particularité. La patiente avait un bon état général. Nous avons évoqué le diagnostic de choriorétinite toxoplasmique active ensuite nous avons demandé un bilan paraclinique pour confirmer notre hypothèse diagnostic ce bilan avait comme résultat: La vitesse de sédimentation: première heure $=16$ millimètres, deuxième heure $=25$ millimètres.La sérologie syphlitique (Venereal Disease Research Laboratory et le Treponema pallidum Haemagglutination Assay) étaient négatives. La sérologie rétrovirale était négative au test Western blot. La sérologie toxoplasmique : les immunoglobulines $\mathrm{G}$ était positive avec un taux de $350 \mathrm{UI} / \mathrm{ml}$ et Immunoglobuline $\mathrm{M}$ positive témoignant d'une infection récente datant moins de 6 mois avant l'examen. Cette sérologie positive de la toxoplasmose a été confirmée par un second prélèvement 3 semaines plus tard par le même laboratoire. L'examen sérologique de l'humeur aqueuse après ponction de la chambre antérieure avait donné un coefficient de Desmonts $\mathrm{C}$ égale à 5 affirmant une production locale d'anticorps et donc le diagnostic de choriorétinite toxoplasmique. La sérologie sanguine de la patiente avait montré également l'absence d'immunoglobulines $G$ et d'immunoglobulines $M$ pour: l'herpès simplex 
virus, le virus varicelle zona, le cytomégalovirus et les candida. Nous avons retenu le diagnostic de choriorétinite toxoplasmique active. La patiente avait été mise sous l'association pyriméthamine et sulfadoxine qui a permis de stopper l'extension du foyer de choriorétinite et de limiter son retentissement sur la fonction visuelle.

\section{DISCUSSION :}

Le diagnostic de la toxoplasmose oculaire est essentiellement basé sur l'examen ophtalmologique $\mathrm{du}$ fond d'œil [1] et les formes cliniques sont nombreuses. Dans notre cas les lésions sont typiques, il s'agit de lésions blanchâtre, rondes, profondes, à bords flou d'un diamètre papillaire de siège temporal supérieur à un et demi de diamètre papillaire de la macula accompagnée d'une tache hémorragique rétinienne au voisinage du foyer. Ces lésions typiques ont été décrites par certains auteurs [1]. Bien que ces signes soit pathognomonique de la toxoplasmose [3]. Cependant les lésions atypiques peuvent exister [4,5]. La toxoplasmose oculaire est rare dans l'enfance [6]. Dans notre cas l'âge de survenu de l'épisode infectieux était de 18 ans. Certains auteurs avaient trouvé un âge moyen de survenu des épisodes initiaux de toxoplasmose oculaire égale à 31,1 ans [7]. Dans notre étude le foyer de choriorétinite était localisé. Certains auteurs avaient noté des formes disséminées [8].

\section{CONCLUSION :}

La choriorétinite toxoplasmique active est une affection liée aux atteintes multiples de la toxoplasmose sa gravité dépend de sa localisation. L'examen du fond d'œil permet de dépister les formes de mauvais pronostic visuelle notamment les localisations papillaire et maculaire. Le retard diagnostic augmente le risque de séquelles visuelles. Le traitement doit être instauré en urgence afin de préserver la fonction visuelle de l'œil atteint.

Conflit d'intérêt : Aucun

\section{REFERENCES :}

1- Bidgoli S, Koch P, Caspers L. Choriorétinite toxoplasmique : PCR positive du vitré malgré une sérologie négative pour toxoplasma gondii. Journal français d'ophtalmologie 2011 ; 34 : 384.e1- 384.e5.

2- Mc cannel CA, Holland GN, Helm CJ, Cornell PJ, Winston JV, Rimmer TG. Causes of uveitis in the general practice of ophthalmology. Am J Ophtalmol 1996; 121:35-45 .

3- Montoya JG, Liesenfeld O. Toxoplasmosis. Lancet 2004; 363: 1965- 76.

4- Fardeau C, Romand S, Rao NA, Cassoux N, Bettembourg O, Thulliez $\mathrm{P}$, et al. Diagnosis of toxoplasmic retinichoroiditis with atypical clinical features. Am J ophtalmol 2002; 134: 196- 203.

5- Theaudin M, Bodhagi B, Cassoux N, Romand S, Le mer Y, Le maitre C, et al. Oxoplasmose oculaire extensive. Conduite diagnostique et thérapeutique. J FR Ophtalmol 2003 ; $26: 921-7$.

6- Pierce EA, D'amico DJ. Ocular toxoplasmosis pathogenesis diagnosis, and management. Semin Ophthalmol 1993; 8:40- 52.

7- Bosch- driessen. LE, Berendschot. TT, Ongkosuwito. JV, Rothova. A. Ocular toxoplasmosis: Clinical features and prognosis of 154 patients. Ophthalmology 2002; 109:869-78.

8- Errera. M.H, Chahed. S , Man. H, Garin Y.J.F, Bergmann. J.F, Gaudric. A, Massin. P. 

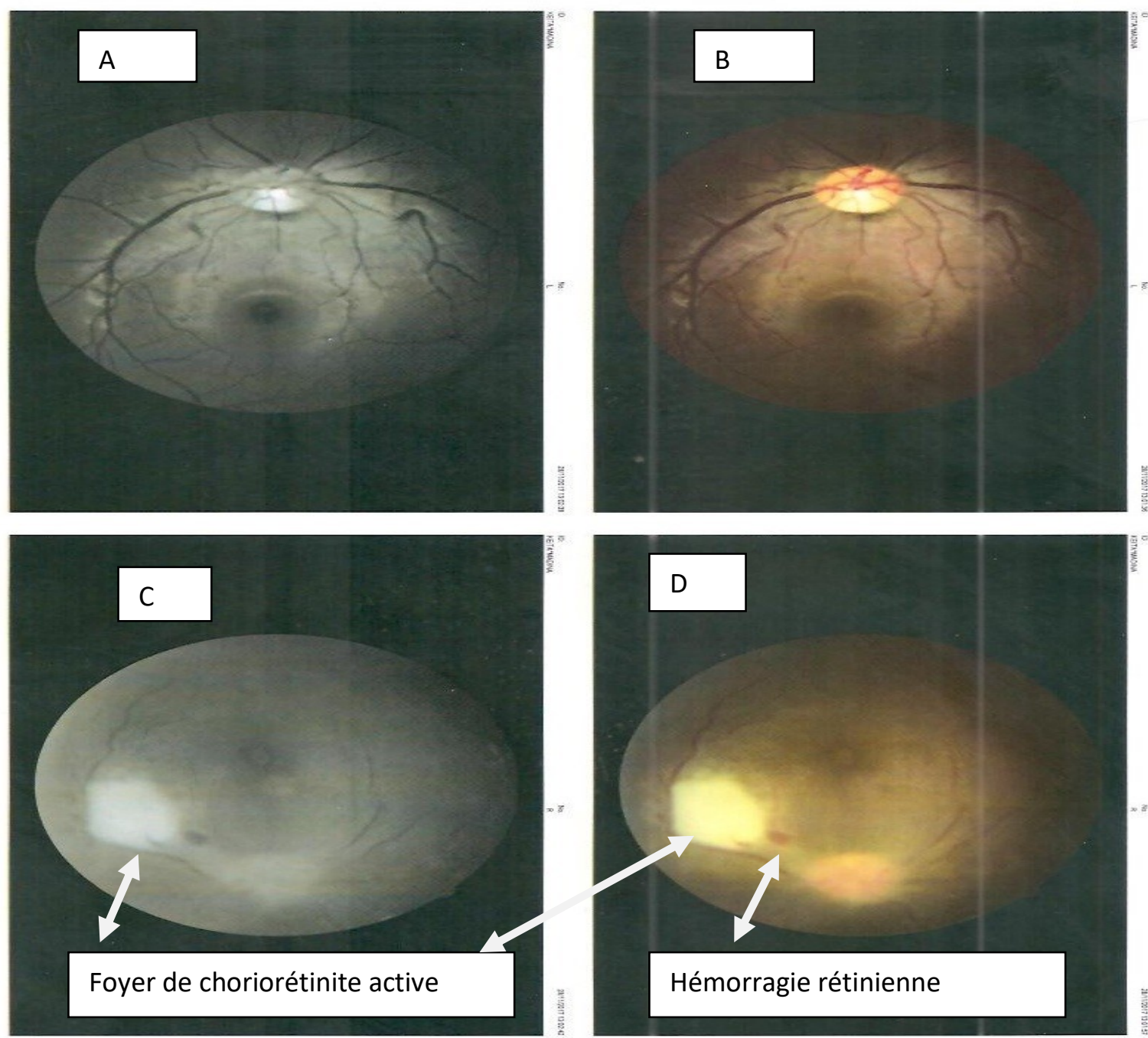

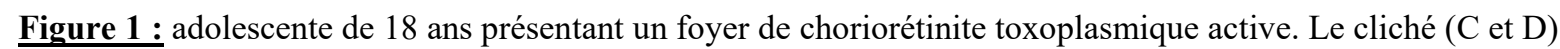
objectivent une lésion blanchâtre, ronde, profonde, à bords flou d'un diamètre papillaire de siège temporal supérieur à un et demi de diamètre papillaire de la macula accompagnée d'une tache hémorragique rétinienne au voisinage du foyer au niveau de l'œil droit alors que le fond œil est normale à l'œil gauche (cliché A et B). 